\title{
Transparent pentacene-based photoconductor: High photoconductivity effect
}

\author{
A. El Amrani, *B. Lucas, F. Hijazi and M. Aldissi \\ XLIM UMR 6172 - Université de Limoges/CNRS \\ 123 avenue Albert Thomas - 87060 Limoges Cedex, France \\ *Corresponding authors: e-mail: bruno.lucas@unilim.fr
}

\begin{abstract}
In this paper, the fabrication and characterisation of pentacene-based photoconductors using indium tin oxide electrodes obtained by ion beam sputtering are discussed. The photoelectric properties of pentacene under red $(632 \mathrm{~nm})$ and ultraviolet $(365 \mathrm{~nm})$ illuminations were investigated. We have shown that the photocurrent was dependent on the wavelength, bias voltage and illumination side of the device. Moreover, we have demonstrated with transparent electrodes that the top contact configuration yields better performance compared to the bottom contact configuration. We obtained a maximum photoconductivity gain of approximately $3 \times 10^{3}$ and a faster dynamic response when the photoconductor with top contact geometry was illuminated with ultraviolet light from the semiconductor side (top illumination), with a photoconductivity estimated at $10^{-4} \Omega^{-1} \mathrm{~cm}^{-1}$.
\end{abstract}

Keywords: Pentacene, UV-visible illumination, Photoconductivity, Photocurrent, Response time.

\section{Introduction}

Ultraviolet (UV)-Visible organic photodetectors have been widely studied due to their potential applications [1-4]. Pentacene (Pn) is a photoconductor which exhibits a small optical band gap and can thus be used for UV and visible photodetection. As a result, the material has been investigated for use in optoelectronic devices such as photodetectors, light activated memory devices, amplifiers or switches controlled by light excitation and optical transducers [5-7]. Organic photoconductors are viable candidates in the area of semiconductor detectors because they combine light detection and signal amplification properties in the simple device.

We have investigated the photoelectric properties, such as photocurrent gain and response time at various bias voltages, of an ITO (Indium Tin Oxide)/Pn/ITO planar structure. The device was illuminated at $632 \mathrm{~nm}$ and $365 \mathrm{~nm}$ from the glass substrate side or the opposite side with bottom contact (BC) and top contact (TC) electrode configurations. 


\section{Experimental}

Schematics of TC and BC photodetector devices we fabricated and their corresponding photoelectric properties are shown in Figure 1 and Figure 2 respectively. The ITO electrodes are obtained by ion beam sputtering with high conductivity $\left(10^{3} \Omega^{-1} \mathrm{~cm}^{-1}\right)$ and transmittance (90\%) [8]. Two ITO contacts (separated by $100 \mu \mathrm{m}$ and defined as the channel) are used to apply a bias voltage to the photoconductor. The pentacene films $(50 \mathrm{~nm})$ were obtained by vacuum evaporation without heating the substrate with a deposition rate of $1 \AA / \mathrm{s}$.

All the devices are fabricated on glass substrates and tested at room temperature in open air. The static and dynamic characteristics of the photoconductor were carried out using computer-controlled 4200 SMU Keithley equipment. A He-Ne laser (632 nm) and a UV lamp (model B 100 AP UVP with emission centred at $365 \mathrm{~nm}$ ) with a power of approximately 7 $\mathrm{mW} / \mathrm{cm}^{2}$ situated at $25 \mathrm{~cm}$ from the photoconductor active layer, were used to irradiate the device from either side of the device, "top illumination" or "bottom illumination". An atomic force microscope (AFM) was used to characterize the topography.

\section{Results and discussion}

Photoconductivity corresponds to an increase in the electrical conductivity $(\sigma)$ of a semiconductor under illumination. Two conditions must be fulfilled: (i) the photon energy $(h v)$ must be higher than the material gap energy $\left(E_{g}\right)$ so that an electron passes from the highest occupied molecular orbital (HOMO) band to the lowest unoccupied molecular orbital (LUMO) band; consequently, excess free carriers will contribute to the increase in electrical conductivity; and (ii) the absorption coefficient $(\alpha)$ of the material must be sufficiently small so that the light can contribute efficiently to the generation of the charge carriers in the bulk of the device channel.

Carrier generation in the allowed bands of pentacene is possible since the energy of the emitted light is $1.96 \mathrm{eV}(632 \mathrm{~nm})$ and $3.4 \mathrm{eV}(365 \mathrm{~nm})$ for red and UV illumination, respectively, which are higher than the gap of pentacene $\left(\mathrm{E}_{\mathrm{g}} \approx 1.8 \mathrm{eV}\right)$ [9]. Moreover, the injection of holes from ITO into the HOMO band of pentacene is favored, due to the low energy barrier which is approximately $0.2 \mathrm{eV}$ [9] at the ITO/pentacene interface.

Figure 1 shows the photocurrent-voltage (I-V) characteristics of the fabricated photoconductor with $\mathrm{BC}$ configuration. We note a quasi-linear photoelectric response with the applied voltage, due to the alignment of the ITO Fermi level with the HOMO band of Pn, 
indicating that the ITO/pentacene contact is ohmic. The dark current is $\approx 10^{-10} \mathrm{~A}$ at $-100 \mathrm{~V}$ bias voltage. Upon red and UV top illumination, the photocurrent increases to $5 \times 10^{-10} \mathrm{~A}$ (Fig. 1a) and $27 \times 10^{-10} \mathrm{~A}$ (Fig. 1b) due to the photogeneration of electron-hole pairs in the semiconductor, which results in a photocurrent gain of 5 and 27, respectively.

For red and UV bottom illumination, the photocurrent gain remains at approximately 9. At $632 \mathrm{~nm}$, the photocurrent is higher in the case of bottom illumination where light produces more free carriers in the bulk of the active layer, and thus a better enhanced photocurrent than top illumination, which could be due to charge carrier detrapping processes in the semiconductor channel being more effective. However, at $365 \mathrm{~nm}$, the maximum photocurrent is obtained for top illumination, which corresponds to an antibatic effect $[10,11]$, where the photocurrent is inversely proportional to the absorption coefficient $(\alpha)$ of pentacene. A previous study [12] has shown that $\alpha(365 \mathrm{~nm})=1 / 2 \alpha(632 \mathrm{~nm})$.

Figure 2 shows the I-V characteristics of the photoconductor with TC electrodes. A linear behaviour of both dark current and photocurrent is once more observed. The dark current remains around $10^{-10} \mathrm{~A}$ at $-100 \mathrm{~V}$, and upon red and $\mathrm{UV}$ top illumination, the photocurrent increases up to $8.5 \times 10^{-9} \mathrm{~A}$ (Fig. 3a) and $1.2 \times 10^{-7} \mathrm{~A}$ (Fig. 2b), resulting in a gain of 85 and $1.2 \times 10^{3}$ respectively. For bottom illumination however, the photoccurent gain remains around 26 at $632 \mathrm{~nm}$ and 120 at $365 \mathrm{~nm}$. We note that the photocurrent gain is much higher than in the previous case (BC configuration) for the same side of illumination. This could be due to the high trap density at the BC electrode interface. In fact, the pentacene film grown on ITO (Fig. 3b) is characterized by a small grain morphology with many grain boundaries which form trapping sites compared to pentacene deposited on glass (Fig. 3a), and consequently affect the charge carrier transport in $\mathrm{BC}$ electrode configuration. Another possible explanation for the very high photocurrent gain in TC configuration and top illumination is that the transparent ITO electrode is used as an optical window [13] for the photons, which allows for higher photogenerated carrier density, and consequently enhanced photocurrent.

Furthermore, we note that the UV radiation is more effective for carrier photogeneration than the red excitation due to the lower absorption coefficient of pentacene at $365 \mathrm{~nm}$ than at $632 \mathrm{~nm}$ [12], which leads to a light penetration depth increase and consequently an increase of the photocurrent.

In the case of one-carrier transport, photoconductivity $(\Delta \sigma)$ is given by [14]:

$$
\Delta \sigma=q p_{0} \Delta \mu_{p}+q \mu_{p} \Delta p
$$


where $\mathrm{q}$ is the elementary charge, $p_{0}$ and $\mu_{p}$ are the values of the hole concentration and mobility in the dark respectively, $\Delta p$ and $\Delta \mu_{p}$ represent the changes due to photoconductivity in hole density and mobility respectively.

Thus, assuming that there is no significant change in the carrier mobility under UV excitation, as shown in our previous work [12], the above equation becomes:

$$
\Delta \sigma=q \mu_{p} \Delta p
$$

According to Ohms law, the photocurrent gain can thus be expressed by:

$$
G=\frac{\mathrm{I}_{\mathrm{illum}}}{\mathrm{I}_{\mathrm{dark}}} \approx \frac{\Delta \sigma}{\sigma},
$$

where $\mathrm{I}_{\text {illum }}$ is the total current under illumination and $\mathrm{I}_{\text {dark }}$ is the dark current.

With conductivity of pentacene being approximately $10^{-7} \Omega^{-1} \mathrm{~cm}^{-1}$, the last equation yields a $\Delta \sigma$ of approximately $10^{-4} \Omega^{-1} \mathrm{~cm}^{-1}$ under UV top illumination.

As the best results in term of photocurrent gain are obtained with a TC configuration and top illumination, we measured the photocurrent transient profiles for both UV and red wavelengths.

Figure 4a shows the photocurrent transient profiles at $632 \mathrm{~nm}$ from $-50 \mathrm{~V}$ to $-200 \mathrm{~V}$. The equilibrium regime is attained rapidly when the majority carrier transit time is high ( $\mathrm{V}=-50$ $\mathrm{V})$. The transit time is inversely proportional to $\mathrm{V}$ because charge carrier recombination is favored. When the light is switched OFF (after 60 seconds of illumination), current relaxation is consistent with an exponential decay, and a persistent photocurrent beyond 60 seconds, which indicates slow recombination of the charge carriers. This remanent photocurrent is also associated with the lifetime of minority carriers (electrons for pentacene), which leads to relaxation to the initial state.

Figure $4 \mathrm{~b}$ shows the photocurrent versus time data during UV top illumination from $-50 \mathrm{~V}$ to $-200 \mathrm{~V}$. A sharp increase in photocurrent is reached with response times that are shorter at high bias voltages (i.e., $140 \mathrm{~s}-200 \mathrm{~s}$ at $-200 \mathrm{~V}$ ). After the light is switched OFF, the current decays exponentially with time, then attains a steady state after few tens of seconds. Photocurrent decay to its initial state is reached faster for the UV wavelength than for the red one. This indicates that at $365 \mathrm{~nm}$, the recombination processes which occur during the current decay are fast enough so that no photocurrent persists during relaxation. Furthermore, as observed above, the current gain is maximized at high voltages $\left(3 \times 10^{3}\right.$ at $\left.-200 \mathrm{~V}\right)$. 


\section{Conclusion}

We have investigated the photoconductivity phenomenon in the pentacene semiconductor under red $(632 \mathrm{~nm})$ and ultraviolet $(365 \mathrm{~nm})$ illumination in a simple pentacene based photoconductor (using ion beam sputtered ITO electrodes). We have shown that the static and dynamic photocurrent characteristics were dependent on wavelength, bias voltage and illumination side of the device. We obtained a photoconductivity of $10^{-4} \Omega^{-1} \mathrm{~cm}^{-1}$, a maximum photoconductivity gain of approximately $10^{3}$ and a faster dynamic response when the photoconductor with top contact geometry was illuminated with UV from the semiconductor side "Top illumination". These promising results indicate that the materials could find use in transparent organic phototransistor applications.

Acknowledgments: The authors thank Mrs Valérie Coudert (SPCTS - UMR 6638Université de Limoges/CNRS -123 avenue Albert Thomas - 87060 Limoges Cedex) for the AFM 2D photos.

\section{References}

1. Q. Tang, L. Li, Y. Song, H. Li, W. Xu, Y. Liu, W. Hu, Yu. Liu, D.Zhu, Adv. Mater. 19, $2624(2007)$

2. J. Xue, S. R. Forrest, Appl. Phys. Lett. 82, 136 (2003)

3. P. Peumans, V. Bulovic', S. R. Forrest, Appl. Phys. Lett. 76, 3855 (2000)

4. S. S. Kim, S. P. Park, J. H. Kim, S. Im, Thin Solid Films 19, 420 (2002)

5. G. Dong, Y. Hu, C. Liang, L. Wang, Y. Qui, Appl. Phys. Lett. 88, 051110 (2006)

6. S. Dutta, K. S. Narayan, Adv. Mater. 16, 2151 (2004)

7. Y.-Y. Noh, K. Yase, and D.-Y. Kim, J. Appl. Phys. 98, 074505 (2005)

8. B. Lucas, W. Rammal, A. Moliton, Eur. Phys. J. Appl. Phys. 34, 179 (2006)

9. K. Kim, Y. K. Yoon, M. -O. Mun, S. P. Park, S. S. Kim, S. Im, J. H. Kim, J. Supercond. 15, $595(2002)$

10. D. Kotowski, B. Kutrzeba-Kotowska, M. Obarowska, R. Signerski, J. Godlewski, Organic Electronics 6, 193 (2005)

11. S. Dutta, K. S. Narayan, Appl. Phys. Lett. 87, 193505 (2005).

12. A. El Amrani, B. Lucas, A. Moliton, Eur. Phys. J. Appl. Phys. 41, 19 (2008)

13. J.-M. Choi, K. Lee, D. K. Hwang, J. H. Kim, S. Im, J. H. Park, E. Kim, J. Appl. Phys. 100, $116102(2006)$

14. R-H. Bube, Photoelectronic propreties of semiconductors (Cambridge Univ. Press, 1992). 


\section{Figure Captions}

Figure 1: Photocurrent-voltage characteristics of pentacene with $\mathrm{BC}$ electrodes tested in the dark and under (a) red illumination $(632 \mathrm{~nm})$ and (b) UV illumination (365 nm)

Figure 2: Photocurrent-voltage characteristics of pentacene with TC electrodes tested in the dark and under (a) red illumination $(632 \mathrm{~nm})$ and (b) UV illumination $(365 \mathrm{~nm})$

Figure 3: $(2 \mu \mathrm{m} \times 2 \mu \mathrm{m})$ AFM patterns of a pentacene film $(50 \mathrm{~nm})$ deposited on glass (a) and ITO (b)

Figure 4: Dynamic photocurrent response of pentacene versus time with TC electrodes at bias voltages of -50 to $-200 \mathrm{~V}$ at (a) $632 \mathrm{~nm}$ and (b) $365 \mathrm{~nm}$. 

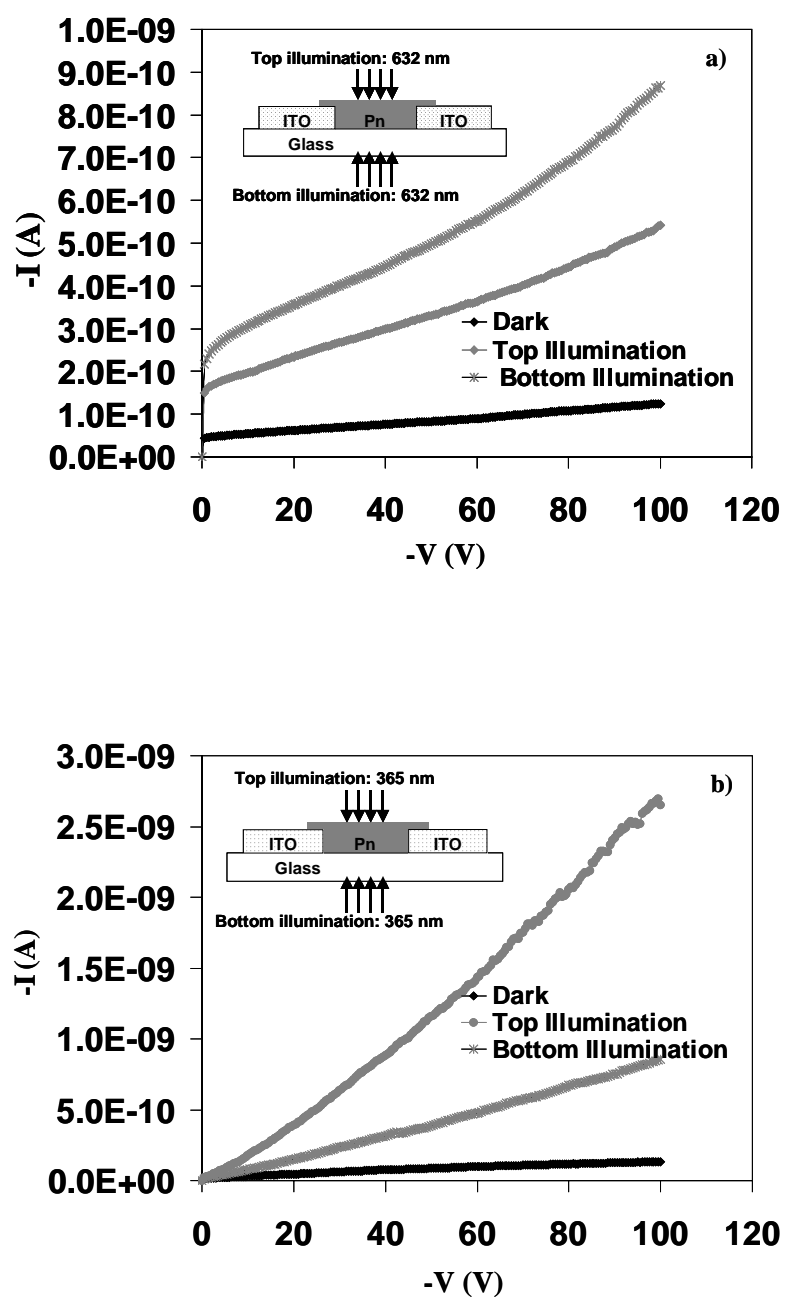

Figure 1 

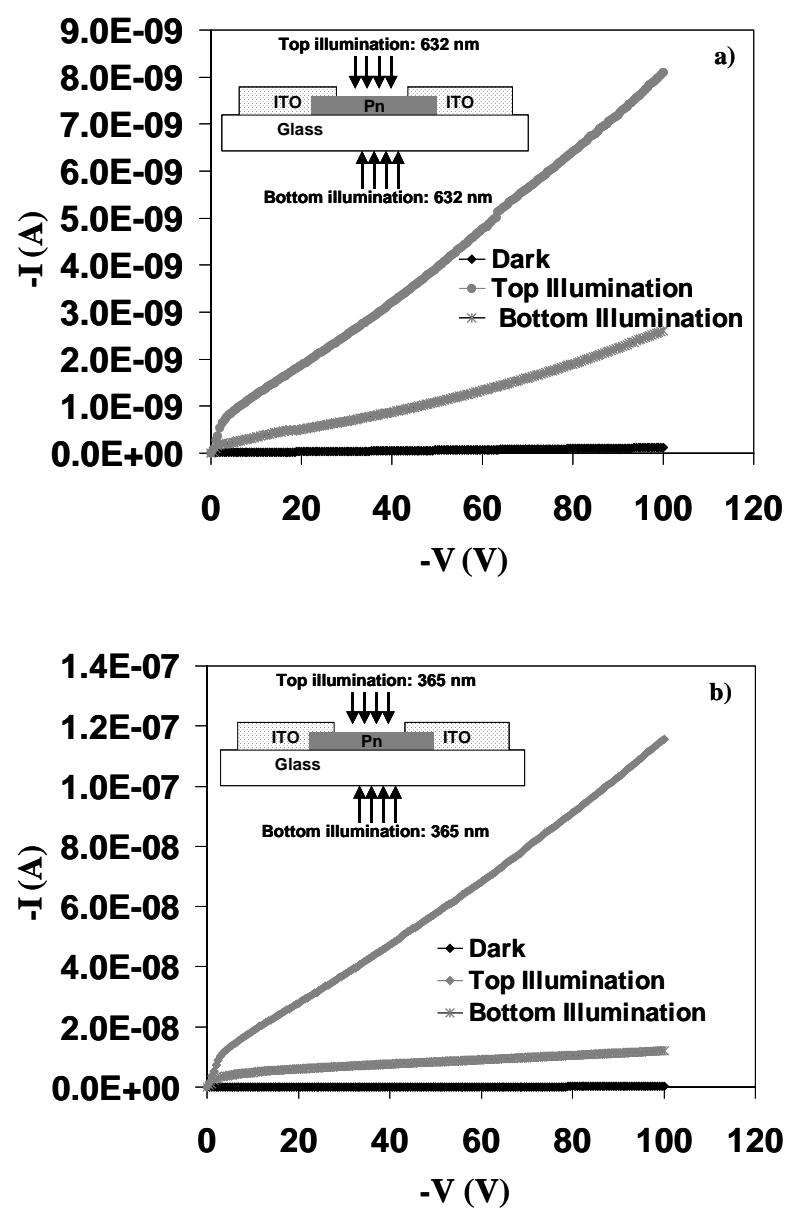

Figure 2 

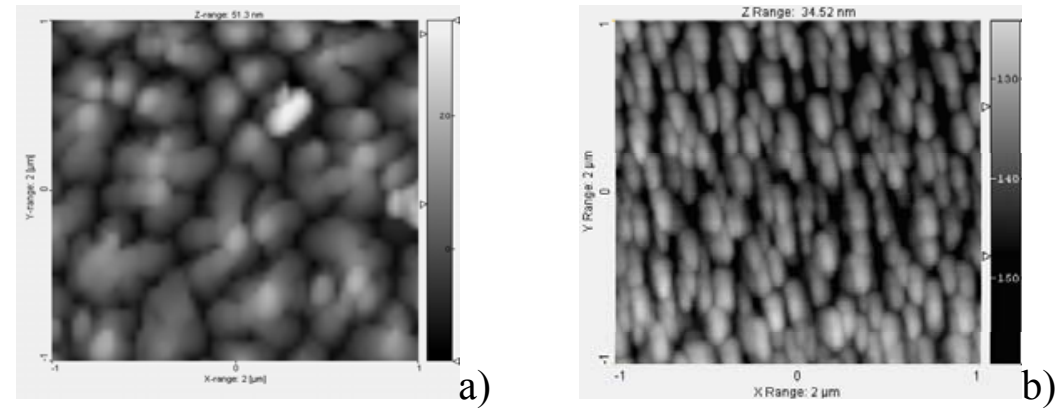

Figure 3 


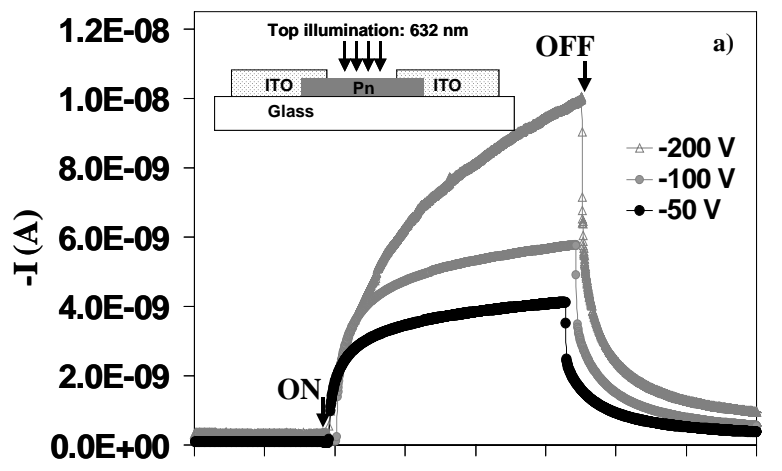

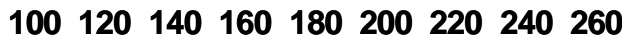

Time (s)

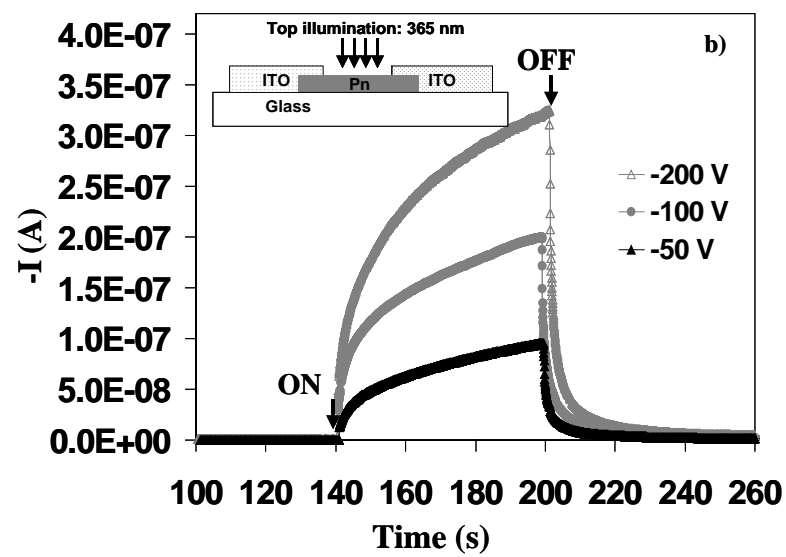

Figure 4 